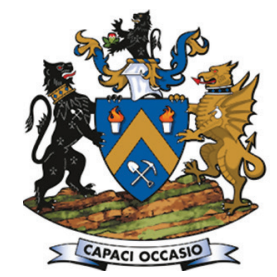

Affiliation:

1 Department of Chemical Engineering, University of KwaZulu-Natal, South Africa.

2 The Centre for Minerals Research, University of Cape Town, South Africa.

\section{Correspondence to:}

I. Govender

Email:

indresan.govender@gmail.com

\section{Dates:}

Received: 25 May 2020

Revised: 9 Nov. 2020

Accepted: 13 Jan. 2021

Published: May 2021

\section{How to cite:}

Ahmed, Elbasher M.E.,

Govender, I., and Mainza, A. 2021

The geometric axial surface

profiles of granular flows in rotating drums.

Journal of the Southern African Institute of Mining and Metallurgy, vol. 121, no. 5, pp. 261-266.

DOI ID:

http://dx.doi.org/10.17159/2411$9717 / 1228 / 2021$

\title{
The geometric axial surface profiles of granular flows in rotating drums
}

\author{
Elbasher M.E. Ahmed ${ }^{1}$, I. Govender ${ }^{1}$, and A. Mainza ${ }^{2}$
}

\section{Synopsis}

A mechanistic description of axial segregation in rotating drum flows remains an open question. Consequently, optimal mixing of grinding balls and rocks for efficient breakage, maximum production of fines, and slurry transport is seldom achieved. Experimental and numerical studies of granular mixtures in rotating drums identify alternating axial bands that eventually coarsen in the long-term limit. Most models of axial segregation are limited to binary mixtures and cannot always predict the logarithmic coarsening effects observed experimentally. A key missing factor is a robust description of the axial free surface profile that is valid across a wide range of flow regimes. We present a practical model of the axial free surface profile by linking it to readily-derived geometric features of the cross-sectional S-shaped free surface profile. A parametric study shows good agreement with experimental measurements reported in the literature and heuristically valid trends.

\section{Keywords}

rotating drum, granular flow, axial profile, comminution, mixing, segregation.

\section{Introduction}

Over the past few decades, rotating drums have attracted considerable research effort by both the engineering and physics communities. The prototypical configuration of rotating drums is often favoured for its ability to display a wide range of granular flow phenomena such as avalanching, chaotic mixing, and segregation (Seiden and Thomas, 2011; Pignatel et al., 2012). Consequently, the processes of blending, mixing, and grinding of materials such as cement, fertilizer, chemicals, and pharmaceuticals are commonly carried out in rotating cylinders (Perry and Green, 1984). In the chemical and process industries, rotating drums are extensively used as mixers, dryers, granulators, and reactors for processing granular materials (Santomaso et al., 2003).

Rotating drum flows exhibit both azimuthal and axial flows with their interplay leading to complex multidirectional flow fields that resist a coherent theoretical description. While cross-sectional flow has been widely studied (Zik et al., 1994; Rajchenbach, 1990); Yamane et al., 1998; Taberlet et al., 2006), axial flow and geometry is rarely discussed (Chou and Lee, 2009; Dury et al., 1998). Chou and Lee (2009) experimentally studied cross-sectional and axial flow patterns of dry granular material in 2D and 3D rotating drums. They argued that sidewall and arching effects lead to a slightly arced axial surface profile and a relatively flat free surface profile in the rolling Froude regime. With increased rotational speeds, the axial surface curvature becomes significant while the free surface profile takes on a distinct S-shape.

Figure 1 is a schematic of the axial bed profile and S-shaped free surface for a drum operating in the cascading Froude regime. To quantify the axial profile of the bed, Chou and Lee (2009) proposed a semi-empirical formula that varies parabolically with axial position, and depends on both the elevation of the surface at axial midpoint $y_{c}$ (see Figure 1) and a coefficient $a_{z}$ that characterizes the axial surface curvature. Unfortunately, these coefficients render their formula impractical in the context of industrial systems that are opaque to visual measurement and too aggressive for sensitive in-situ measurement sensors.

Numerical simulation by the discrete element method (DEM) facilitated a similar study by Dury et al. (1998). To quantify the characteristic length of boundary effects $\xi$, Dury et al. (1998 used, in batch simulations with mono-sized spheres, abstract constants that can become very difficult, if not impossible, to quantify in realistic industrial systems like tumbling mills.. The numerically determined variation of the time-averaged axial repose angle with axial position was fitted to an exponential 


\section{The geometric axial surface profiles of granular flows in rotating drumsin rotating drums}

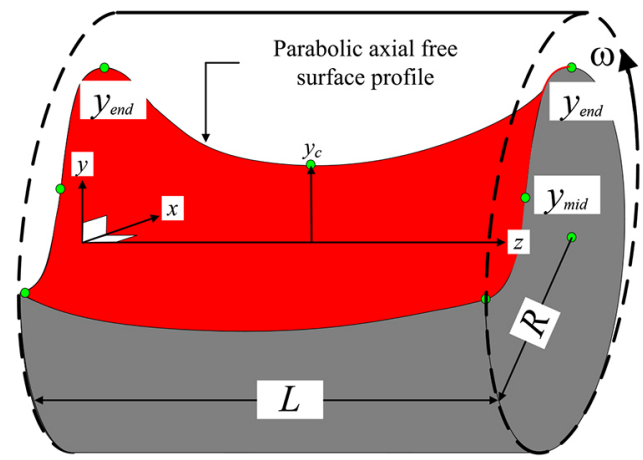

Figure 1-Schematic of axial surface profile (red surface) in a drum with length $L$ and radius $R$ rotating at angular speed $\omega$. The shoulder $y_{\text {end }}$ and midpoint $y_{\text {mid }}$ located on the azimuthal free surface denote vertical heights that are used in the new model given by Equation [2]. We note that $y_{\text {end }}$ and $y_{\text {mid }}$ are easily extracted from the numerical solution of the free surface

function involving the bed repose angle at the axial midpoint and axial boundary wall, $\xi$, and drum length $L$. Notwithstanding the good agreement with DEM simulations, the model cannot be easily used outside of DEM or idealized experimental contexts where $\xi$ is readily quantified. Noting the limitations of the models by Chou and Lee (2009) and Dury et al., (1998), in the present work we propose a new formula that circumvents the need for abstract constants that can become very difficult, if not impossible, to quantify in realistic industrial systems like tumbling mills.

In the next section we describe the construction of the new axial surface profile model. A parametric study then follows, wherein we consider the influence of drum angular speed across the three dominant Froude regimes (rolling, cascading, and cataracting), drum length, and fill fraction on the axial free surface profile. Noting that our model naturally builds upon well-established azimuthal free surface profiles reported in the literature (Zik et al., 1994), these are also included in the parametric study. Finally, the structural similarity between the model by Chou and Lee (2009) and ours allowed us to incorporate their experimentally verified model into the existing analysis by back-fitting the difficult-to-measure axial surface curvature $a_{z}$ to our results.

\section{Construction of the axial-profile}

Chou and Lee (2009) developed a semi-empirical formula to describe the axial surface profiles as parabolic curves along the drum axis (z-axis). Their formula is given by:

$$
y(z)=y_{c}+\frac{a_{z}}{2}\left(z-\frac{L}{2}\right)^{2}
$$

where $y_{c}$ is the surface height at the axial centre-line (see Figure. $1), a_{z}$ is the axial surface curvature (obtained by measurement), and $L$ is the length of the drum. An obvious limitation relates to the difficulty in estimating the constants $y_{c}$ and $a_{z}$ in realistic scenarios, where measurement of these quantities is impractical due to the harsh and opaque environments typically encountered in industrial tumbling mills. To this end we propose a variation on their formula with a view to replacing these constants with simple and easy-to-calculate expressions based on the S-shaped free surface profile. The proposed modification circumvents the need for explicit knowledge of the axial surface curvature, $a_{z}$ and gives the following result for the axial free surface profile.

$$
y(z)=\left(y_{\text {mid }}+R\right)+\frac{y_{\text {end }}-y_{\text {mid }}}{A}\left(z-\frac{L}{2}\right)^{2}
$$

where $A=2 \varpi R L$ is the azimuthal surface area of the drum with radius $R$. The underlying philosophy of our model is that values for $y_{\text {end }}$ and $y_{\text {mid }}$ (see Figure 2) can be obtained from a numerical solution of the differential equation governing the S-shape of the azimuthal free surface. In this regard, several models for the cross-sectional free surface profile exist (Zik et al., 1994; Rajchenbach, 1990); Taberlet et al., 2006). For example, the model of Zik et al. (1994) is given by

$$
\Lambda\left[\frac{d Y_{f s}}{d x}-\mu\right]\left[1+\left(\frac{d Y_{f s}}{d x}\right)^{2}\right]=1-\left[\left(\frac{x}{R}\right)^{2}+\left(\frac{Y_{f s}}{R}\right)^{2}\right]
$$

where $P_{0}$ represents the lithostatic pressure at the bottom of flowing layer, $\left(x, Y_{f s}\right)$ are the coordinates to the free surface, and $\Lambda$ is a lumped parameter given by

$$
\Lambda=\frac{2 P_{0}^{3}}{3 \eta \omega \rho^{2} g^{2} R^{2}}
$$

that depends on the granular viscosity $\eta$, angular speed of the drum $\omega$, bulk density $\rho$, and drum radius $R$. Figure 2 illustrates a free surface solution for the parameters given in Table I.

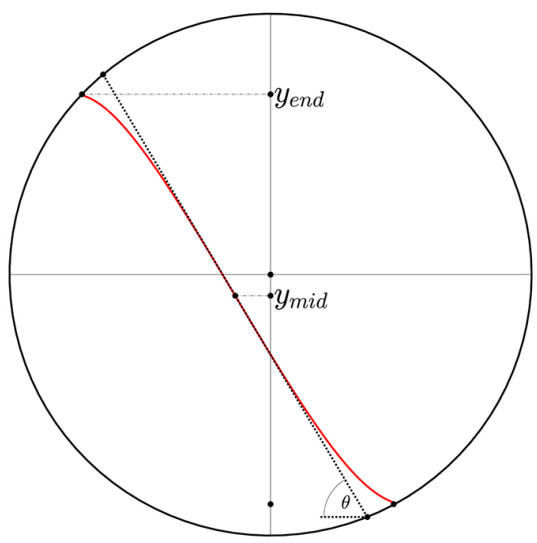

Figure 2-S-shaped free surface profile (red curve) based on the values in Table I. The shoulder position $y_{\text {end }}$ and inflection point $y_{\text {mid }}$, obtained from

\begin{tabular}{|c|c|c|}
\hline Parameter & Description & SI Units \\
\hline$\rho=2500$ & Density & $\frac{g}{m^{3}}$ \\
\hline$g=9: 81$ & Local gravitational acceleration & $\frac{m}{s^{2}}$ \\
\hline$R=0: 238$ & Drum radius & $\mathrm{m}$ \\
\hline $\begin{array}{l}P_{\text {crit }}=60 \\
\eta=6 \quad 10^{-3}\end{array}$ & $\begin{array}{c}\text { Percentage of critical speed } \\
\text { Granular viscosity }\end{array}$ & $\begin{array}{c}\% \\
\mathrm{~Pa} \cdot \mathrm{s}\end{array}$ \\
\hline$d=3 \quad 10^{-3}$ & Particle diameter & $\mathrm{m}$ \\
\hline$h_{0}=1: 3 d$ & Depth of owing layer & $\mathrm{m}$ \\
\hline $\begin{array}{l}\phi=0: 58 \\
\theta=40\end{array}$ & Average solids fraction & $\begin{array}{l}{[-]} \\
\text { deq }\end{array}$ \\
\hline$P_{0}=\rho \phi g h 0 \cos \left(\theta_{0}\right)$ & Pressure at depth ho & $\mathrm{Pa}$ \\
\hline$\alpha=0: 4$ & Fill fraction & {$[-]$} \\
\hline$L=2 R$ & Drum length & {$[m]$} \\
\hline$\mu=0: 4$ & Mohr-Coulomb friction coecient & {$[-]$} \\
\hline
\end{tabular}
this numerical solution, form the key inputs to the model given by Equation [2]. The origin of the $(x, y)$ coordinate system is located at the centre of the drum 


\section{The geometric axial surface profiles of granular flows in rotating drumsin rotating drums}

By exploiting the symmetry of the azimuthal free surface model, solutions to the inflection point $y_{\text {mid }}=-1.92 \mathrm{~cm}$ and shoulder position $y_{\text {end }}=16.34 \mathrm{~cm}$ were obtained for the configuration depicted in Table I. Substitution into Equation [2] then yields the axial surface profile proposed in this paper. Figure 3 illustrates the axial surface profile using our model, Equation [2], and that of Chou and Lee (2009) Equation [1]. In the absence of measured values for the axial curvature parameters $a_{z}$ and $y_{c}$ required by the model of Chou and Lee (2009), we perform a least-squares fit of Equation [1] against our solution by treating $a_{z}$ and $y_{c}$ as the unknown constants to be fitted. The maximum vertical displacement of the axial surface, denoted $V_{\text {disp }}$ in Figure 3, will serve as a proxy for quantifying the degree of curvature, i.e., the larger $V_{\text {disp }}$, the higher the degree of curvature.

\section{Result and discussion}

Noting that our model is influence by the azimuthal free surface profile (which ultimately determines the values of $y_{\text {mid }}$ and $y_{\text {end }}$ ) and the drum length, we perform a parametric study by varying the drum fill fraction $\alpha$, drum length $L$ and Froude number $F r=\frac{\omega^{2} g}{R}$

\section{Froude number}

To ensure a wide Froude regime coverage, we tested drum speeds consistent with rolling ( $1 \%$ critical speed), cascading (40\% critical speed), and cataracting (85\% critical speed) modes.
Figure 4 shows the influence of drum speed, expressed as a percentage of the critical speed, on the azimuthal and axial free surface profiles. At $1 \%$ of critical speed (Figures. $4 \mathrm{a}$ and $4 \mathrm{~b}$ ), the azimuthal free surface is flat while the axial free surface is slightly curved with $V_{\text {disp }}<1 \mathrm{~cm}$. At $40 \%$ critical speed (Figures $4 \mathrm{c}$ and $4 \mathrm{~d}$ ), an increase in the repose angle, a slightly S-shaped azimuthal free surface profile, and an increased curvature in the axial free surface profile are evident, with $V_{\text {disp }}<1.5 \mathrm{~cm}$. At a fully cataracting speed corresponding to $85 \%$ of critical, (Figures $4 \mathrm{e}$ and $4 \mathrm{f}$ ), the azimuthal free surface depicts the highest bed repose, a slightly more S-shaped azimuthal free surface than

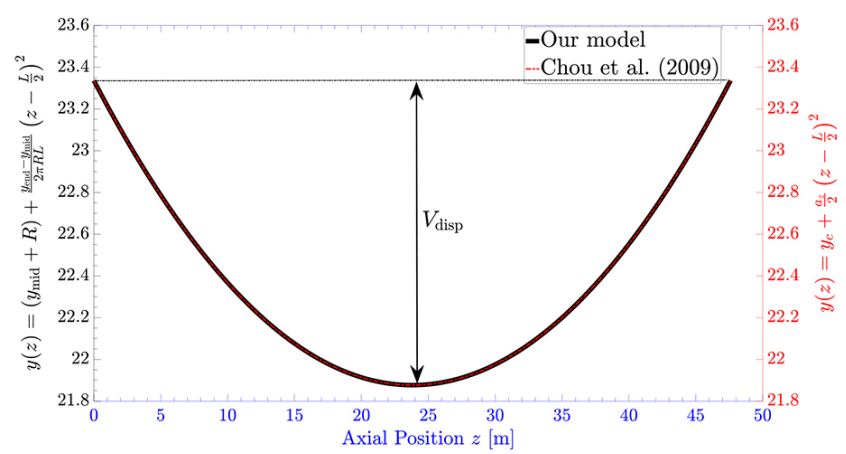

Figure 3-Axial surface profiles based on the data given in Table I. In the absence of measured values, $y_{c}$ employed in Equation [1] is obtained using a least-squares fit of Equation [1] to the free surface profile in Figure 2

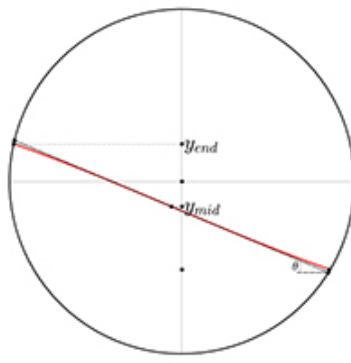

(a)

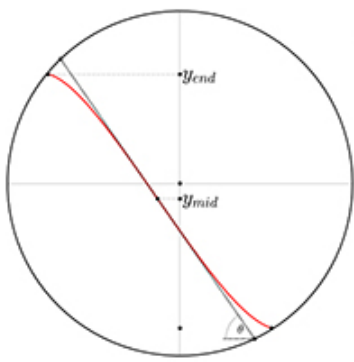

(c)

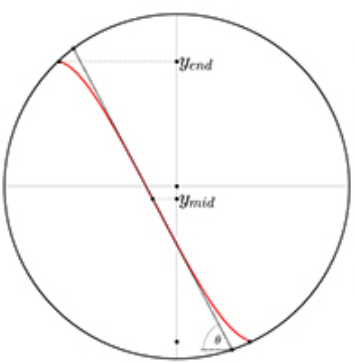

(e)

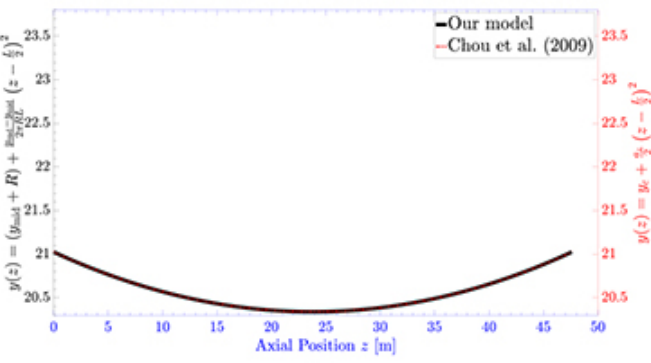

(b)

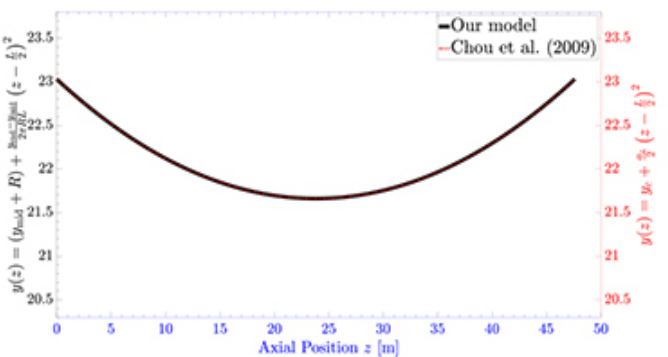

(d)

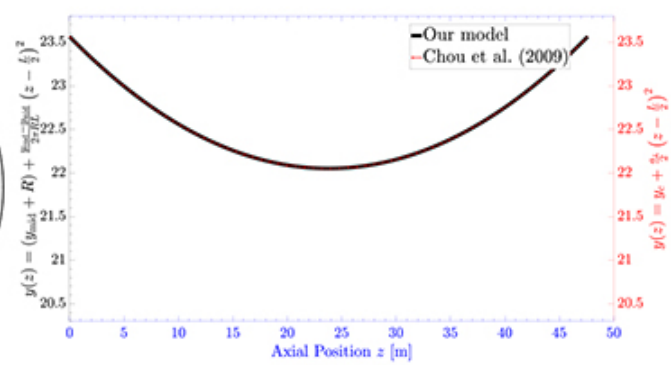

(f)

Figure 4-The effect of drum speed on the azimuthal and axial free surface profiles for the operating conditions specified in Table I. (a, b): 1\% critical speed, (c, d): $40 \%$ critical speed, (e, f): $85 \%$ critical speed 


\section{The geometric axial surface profiles of granular flows in rotating drumsin rotating drums}

the two lower speeds, and the highest curvature in the axial free surface, with $V_{\text {disp }}=1.5 \mathrm{~cm}$. We also note that the trends found are very similar to the experimentally measured surface profiles by Chou and Lee (2009, see Figure. 22d in their paper).

\section{Drum length}

Using the configuration in Table I, we next varied the drum length in multiples of the drum radius $-L=(R, 2 R, 4 R)$; see Figure 5 . While the azimuthal free surface profile does not seem to vary much with drum length (Figures $5 \mathrm{a}, 5 \mathrm{c}, 5 \mathrm{e}$ ), the axial free surface curvature definitely increases with increasing drum length (Figures $5 \mathrm{~b}, 5 \mathrm{~d}, 5 \mathrm{f}$ ). Interestingly, $V_{\text {disp }}$ doubles each time the drum length is doubled, suggesting a one-to-one doubling relationship between the drum length and $V_{\text {disp }}$. Consistent with the findings of Dury et al. (1998), when the drum is sufficiently long such that $L / 2>\xi$, the characteristic length of the boundary effects drops off sharply, leading to a slumped axial midsection of the bed that is characterized by a large value of $V_{\text {disp. }}$. This might be the reason why the curvature follows a doubling relationship as opposed to a linear one.

\section{Fill fraction}

The final parametric study involves the drum fill fraction $\alpha$. Again, using the configuration in Table I, the fill fraction was varied: $\alpha=0.1,0.2,0.4,0.8$. The effect of increasing the drum fill fraction is to simply shift the axial free surface vertically upwards without much influence on $V_{\text {disp }}$. We note here that the insensitivity to fill fraction may be partly due to the limitations of the azimuthal free surface model.

\section{Conclusion}

A model of the axial free surface profile in a rotating drum was developed. While exhibiting a similar structure to the model of Chou and Lee (2009), the proposed model offers significant practical advantages over the former. In this regard, a well-chosen azimuthal free surface model is sufficient to numerically derive the key ingredients for the axial free surface model. A parametric study of the model considered variations in Froude regime, drum length, and fill fraction. The results appear consistent with the experimental findings of Chou and Lee (2009) and the numerical results obtained by Dury et al. (1998). The parametric study spanned operating conditions consistent with comminution practice in minerals processing as regards drum speed $(<85 \%$ of critical), drum aspect ratios $(D: L=2: 1-1: 2)$, and fill fractions ( $\alpha$ $=0.2,0.4$ ); suggesting that the model can be used to complement understanding of segregation and transport in tumbling mills. On the rheological side, the realistic curvature response of the model to drum length suggests that the model inputs $y_{\text {end }}$ and $y_{\text {mid }}$ ) might be good proxies for the axial rheology that ultimately drives flow and shape in granular systems.

Future work will employ other azimuthal-free surface models (Rajchenbach, 1990); Yamane et al., 1998; Taberlet et al., 2006) in a similar parametric study. We also hope to incorporate measured free surface profiles via the technique of positron

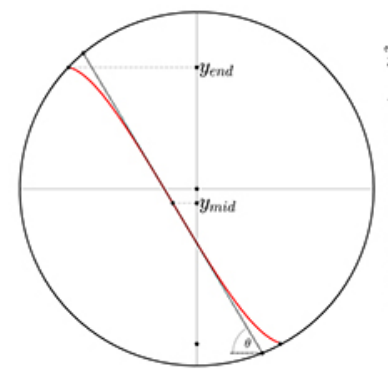

(a)

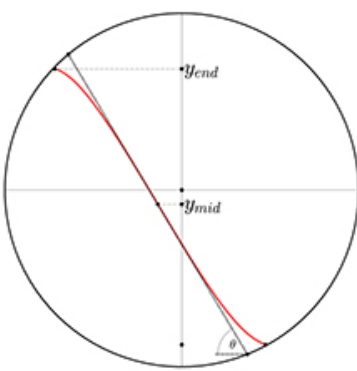

(c)

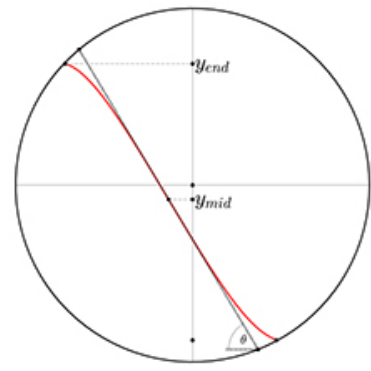

(e)

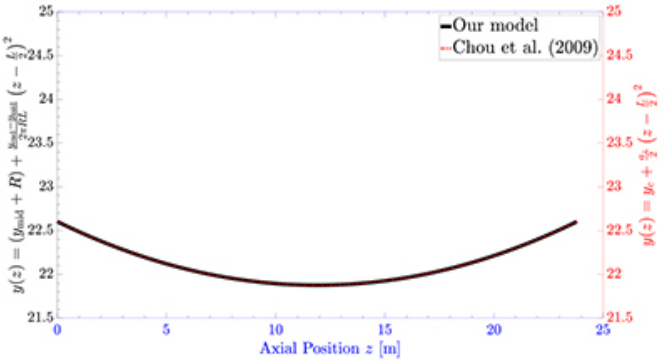

(b)

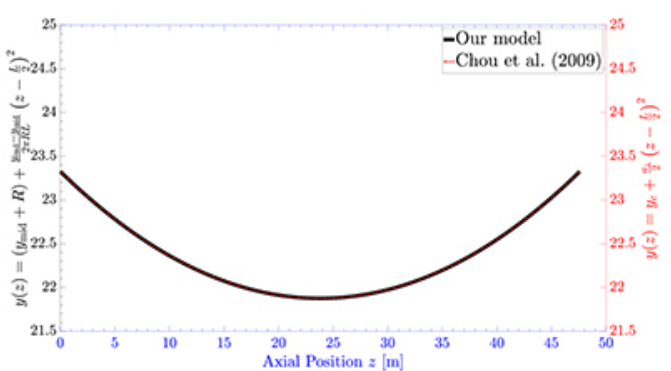

(d)

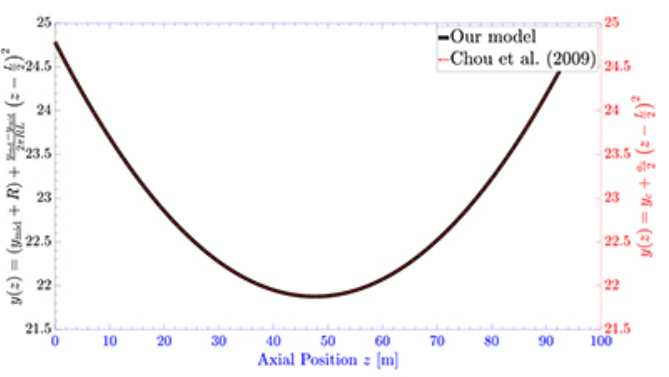

(f)

Figure 5-The effect of drum length $L$ on the azimuthal and axial free surface profiles for the operating conditions specified in Table $I$. (a, b): $L=R$, (c, d): $L=2 R$, (e, $f): L=4 R$ 


\section{The geometric axial surface profiles of granular flows in rotating drumsin rotating drums}

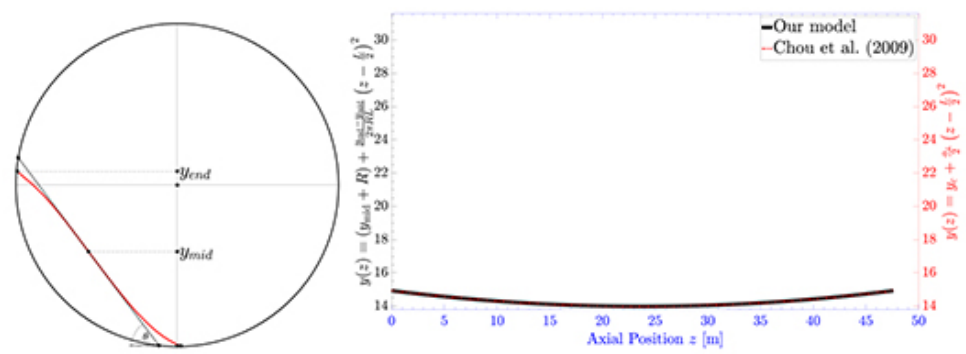

(a)

(b)

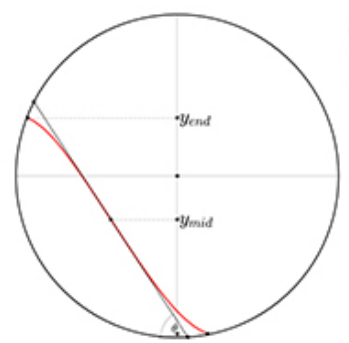

(c)

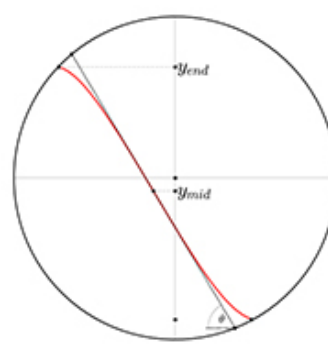

(e)

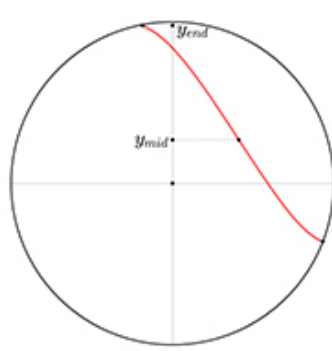

(g)

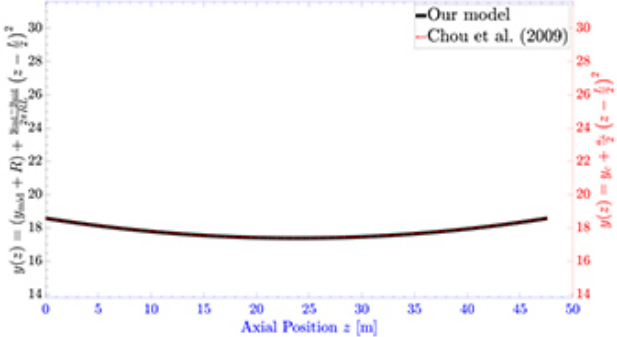

(d)

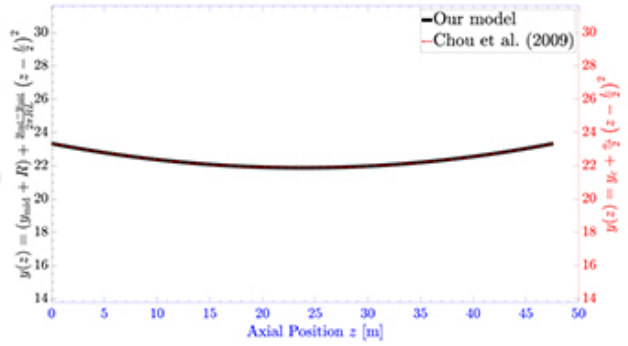

(f)

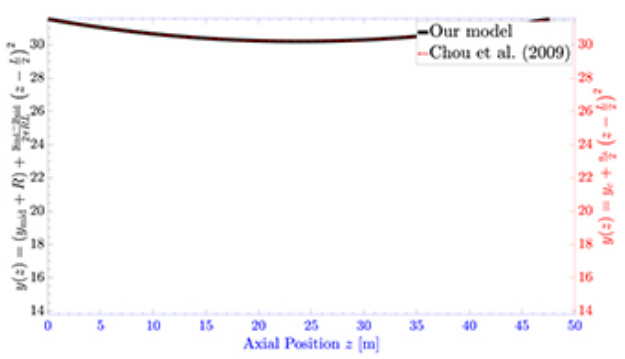

(h)

Figure $6-$ The effect of drum fill fraction $a$ on the azimuthal and axial free surface profiles for the operating conditions specified in Table I. (a, b): $a=0.1,(c, d)$ : $a=0.2,(e, f): a=0.4,(g, h): a=0.8$

emission particle tracking and DEM with a view to building a semi-empirical formula that can be readily used to guide comminution transport optimization.

\section{Acknowledgment}

This work was supported by the Centre for Minerals Research (CMR) in the University of Cape Town Physics Department and the Department of Chemical Engineering at the University of KwaZulu-Natal.

\section{References}

CHou, H-T. and LeE, C-F. 2009. Cross-sectional and axial flow characteristics of dry granular material in rotating drums. Granular Matter, vol. 11, no. 1. pp. 3-32.

DurY, C.M., Ristow, G.H., Moss, J.M., and NAKAgaWA, M. 1998. Boundary effects on the angle of repose in rotating cylinders. Physical Review E, vol. 57. https://doi. org/10.1103/PhysRevE.57.4491

Perry, R.H. and Green, D.W. 1984. Perry's Handbook of Chemical Engineering. Chemical Engineering Series, McGraw Hill,New York.
Pignatel, F. Asselin, C., Krieger, L., Christov, I.C., Ottino, J.M., and Lueptow, R.M. 2012. Parameters and scalings for dry and immersed granular flowing layers in rotating tumblers. Physical Review E, vol. 86, no. 1. pp. 001304-12.

Rajchenbach, J. 1990. Flow in powders: From discrete avalanches to continuous regime. Physical Review Letters, vol. 65, no. 18. pp. 2221-2225.

SAnTomaso, A.C., Ding, Y.L., Lickiss, J.R., and YoRK, D.W. 2003. Investigation of the granular behaviour in a rotating drum operated over a wide range of rotational speed. Transactions of the Institute of Chemical Engineers, vol. 81. pp. 936-945.

SeIDEn, G. and Thomas, P.J. 2011. Complexity, segregation, and pattern formaiton in rotating-drum flows. Reviews of Modern Physics, vol. 83. pp. 1323-1365.

TABerlet, N., Newey, M., Richard, P., and Losert, W. 2006. On axial segregation in a tumbler: An experimental and numerical study. Journal of Statistical Mechanics: Theory and Experiment, vol. 2006. P07013:041302.

Yamane, K., Nakagawa, M., Altobeli, S.A., Tanaka, T., and Tsuj, Y. 1998. Steady particulate flows in a horizontal rotating cylinder. Physics of Fluids, vol. 10 pp. 1419-1427.

Zik, O., Levine, D., Lipson, S.G., Shtrikman, S., and Stavans, J. 1994. Rotationally induced segregation of granular materials. Physical Review Letters, vol. 73. pp. 644-648. 